\title{
UNITAL MULTIPLICATIONS ON A HILBERT SPACE
}

\author{
JOHN FROELICH
}

(Communicated by Palle E. T. Jorgensen)

\begin{abstract}
We provide a new proof of two theorems of Ingelstam that identify the Hilbert spaces with a unital multiplication satisfying $\|x y\| \leq\|x\|\|y\|$ and $\|1\|=1$.
\end{abstract}

Ingelstam [3, Corollary 2], as a consequence of his analysis of the vertex property for Banach algebras with identity, proved that any complex Hilbert space admitting a unital multiplication satisfying

$$
\|x y\| \leq\|x\|\|y\|, \quad\|1\|=1
$$

must be the complex numbers. It was later conjectured by Kaplansky [4] and proved by Ingelstam [4, Theorem 2] that any real Hilbert space admitting a unital multiplication with the above properties must be the real, complex, or quaternion numbers.

The above results are relevant to the theory of strictly cyclic operator algebras $[5,8]$, and our recent interest in these algebras led us to reconsider the proofs. A unital operator algebra $\mathscr{A}$ on a Hilbert space $H$ is called strictly cyclic if there is a vector $h \in H$ such that the linear manifold $\mathscr{A} h=\{A h: A \in \mathscr{A}\}=H$. The algebra is called separated if in addition $A h=0$ implies that $A=0$. If $\mathscr{A}$ is a separated strictly cyclic algebra with strictly cyclic and separating vector $e$ then the map $A \rightarrow A e$ gives a Banach space isomorphism between the operator algebra $\mathscr{A}$ and the Hilbert space $H$. Thus the operator norm on $\mathscr{A}$ is equivalent to an inner product norm \|\| . Therefore we may choose \|\| such that

$$
\|A B\| \leq K\|A\|\|B\|, \quad\|1\|=1
$$

for any $A, B \in \mathscr{A}$, where $K \geq 1$. Conversely, given a Hilbert space $H$ with unital multiplication satisfying the above properties, the left regular representation

$$
\mathscr{A}=\left\{L_{h}: h \in H\right\}, \quad L_{h}(k)=h \cdot k
$$

is a separated strictly cyclic operator algebra with cyclic vector 1 and the operator norm on $\mathscr{A}$ is equivalent to the Hilbert space norm.

Thus separated strictly cyclic operator algebras and Hilbert spaces with continuous unital multiplication are equivalent notions and Ingelstam's results may

Received by the editors June $26,1991$.

1991 Mathematics Subject Classification. Primary 46H20, 47D25.

(C) 1993 American Mathematical Society $0002-9939 / 93 \$ 1.00+\$ .25$ per page 
be interpreted as saying that the operator norm equals the Hilbert space norm only in certain special cases.

In this paper we provide a unified approach to Ingelstam's theorems that relies only on a few elementary ideas from the theory of Banach algebras and Hilbert space geometry.

Theorem 1. Let $H$ be a real Hilbert space with a unital multiplication satisfying

$$
\|x y\| \leq\|x\|\|y\|, \quad\|1\|=1 .
$$

Then $H$ is the real, complex, or quaternion numbers.

Proof. We will show that $H$ is a division ring. If not, then there is a nonzero $a \in H$ that is not invertible. Consider the closed subalgebra $\mathscr{A}$ generated by $a, 1$. There is a maximal ideal $M \subset \mathscr{A}$ containing $a$. Then $\mathscr{A} / M$ is a field isomorphic to $R$ by the map $[\lambda \cdot 1] \rightarrow \lambda$, and we have that $q: \mathscr{A} \rightarrow \mathscr{A} / M \approx R$ is a multiplicative linear functional with $q(1)=1, \operatorname{ker} q=M$, and $\|q\|=1$ [7, Theorem 10.7]. By the Riesz-representation theorem there is a $y \in \mathscr{A}$ such that

$$
q(x)=(x, y) \text { for all } x \in \mathscr{A} .
$$

Since $\|q\|=1$, it follows that $\|y\|=1$ and $q(1)=1=(1, y)$ implies that $y=$ 1 by the equality condition of the Cauchy-Schwarz inequality. It follows from ker $q=M$ that 1 is orthogonal to $M$. Therefore $\mathscr{A}=R \oplus M$ isometrically.

Let $m \in M$ and consider

$$
\left\|(1+m)^{2}\right\|^{2} \leq\left(1+\|m\|^{2}\right)\left(1+\|m\|^{2}\right) .
$$

This yields

$$
2\|m\|^{2} \leq\|m\|^{4}-\left\|m^{2}\right\|^{2}-4\left(m, m^{2}\right) .
$$

Replacing $m$ by $\varepsilon \cdot m$, dividing by $\varepsilon^{2}$, and letting $\varepsilon \rightarrow 0$, we obtain that $\|m\|=0$ so $M=\{0\}$. This is a contradiction. It follows that $H$ is a normed division ring over $R$ and so $H$ is the real, complex, or quaternion numbers [6, Theorem 1.7.6].

The same argument also produces

Theorem 2. Let $H$ be a complex Hilbert space with a unital multiplication satisfying

Then $H=\mathbb{C}$.

$$
\|x y\| \leq\|x\|\|y\|, \quad\|1\|=1 .
$$

\section{ADDED IN PROOF}

After the paper was submitted we discovered that M. F. Smiley [9] has also obtained a simplification of Ingelstam's theorems different from ours.

\section{REFERENCES}

1. H. F. Bohneblust and S. Karlin, Geometrical properties of the unit sphere of Banach algebras, Ann. of Math. (2) 62 (1955), 217-229.

2. J. Froelich, Strictly cyclic operator algebras, Trans. Amer. Math. Soc. 325 (1991), 73-86.

3. L. Ingelstam, $A$ vertex property for algebras with identity, Math. Scand. 11 (1962), 22-32.

4. __ Hilbert algebras with identity, Bull. Amer. Math. Soc. 69 (1963), 794-795. 
5. A. Lambert, Strictly cyclic operator algebras, Pacific J. Math. 39 (1971), 717-726.

6. C. Rickart, General theory of Banach algebras, van Nostrand, New York, 1960.

7. W. Rudin, Functional analysis, McGraw-Hill, New York, 1973.

8. A. Shields, Weighted shift operators and analytic function theory in topics in operator theory, Math. Survey Monographs, vol. 13, Amer. Math. Soc., Providence, RI, 1974.

9. M. F. Smiley, Real Hilbert algebras with identity, Proc. Amer. Math. Soc. 16 (1965), 440441.

Department of Mathematics, University of Houston, Houston, TeXas 77204-3476 